\title{
Simulation of mode conversion at the magnetopause
}

\author{
LIN Yu ${ }^{1,2^{*}}$, JOHNSON Jay $^{3}$, WANG XueYi ${ }^{1} \&$ LU QuanMing ${ }^{2}$ \\ ${ }^{1}$ Physics Department, Auburn University, Auburn 36849, USA; \\ ${ }^{2}$ Department of Geophysics and Planetary Science, University of Science and Technology of China, Hefei 230026, China; \\ ${ }^{3}$ Princeton Plasma Physics Laboratory, Princeton University, Princeton 08543, USA
}

Received November 17, 2011; accepted January 30, 2012

\begin{abstract}
Two-dimensional (2-D) and three-dimensional (3-D) hybrid simulations are carried out for mode conversion from fast mode compressional wave to kinetic Alfvén waves (KAWs) at the inhomogeneous magnetopause boundary. For cases in which the incident fast wave propagates in the $x z$ plane, with the magnetopause normal along $x$ and the background magnetic field pointing along $z$, the 2-D ( $x z$ ) simulation shows that KAWs with large wave number $k_{x} \rho_{i} \sim 1$ are generated near the Alfvén resonance surface, where $\rho_{i}$ is the ion Larmor radius. Several nonlinear wave properties are manifest in the mode conversion process. Harmonics of the driver frequency are generated. As a result of nonlinear wave interaction, the mode conversion region and its spectral width are broadened. In the 3-D simulation, after this first stage of the mode conversion to KAWs with large $k_{x}$, a subsequent generation of KAW modes of finite $k_{y}$ is observed in the later stage, through a nonlinear parametric decay process. Since the nonlinear cascade to $k_{y}$ can lead to massive transport at the magnetopause, the simulation results provide an effective transport mechanism at the plasma boundaries in space as well as laboratory plasmas.
\end{abstract}

mode conversion, magnetopause, wave interaction, kinetic Alfvén wave (KAW)

Citation: Lin Y, Johnson J, Wang X Y, et al. Simulation of mode conversion at the magnetopause. Chin Sci Bull, 2012, 57: 1375-1383, doi: 10.1007/s11434-012-5056-8

Transport of mass, momentum, and energy at plasma boundaries is fundamentally important in the dynamics of space plasmas. The transport at the magnetopause boundary drives much of the magnetospheric dynamics and is therefore of great interest to the magnetospheric research. The co-existence of the solar wind and magnetospheric populations in the magnetopause boundary layer and the thickening of the magnetopause boundary layer during periods of northward interplanetary magnetic field (IMF) indicates the existence of efficient transport processes across the magnetopause [1-10]. Wave-particle diffusive processes associated with large-amplitude ULF waves, which are frequently observed at the magnetopause boundary, are believed to provide one of effective mechanisms of plasma entry at the magnetopause [11-29].

It has been suggested that mode conversion from compressional to Alfvén modes provides a source of Alfvén waves at

*Corresponding author (email: ylin@ physics.auburn.edu) the magnetopause boundary [30-35]. Multi-point measurements have verified that the dispersion of the broadband waves is consistent with the kinetic Alfvén waves (KAWs) $[35,36]$. Such mode conversion may lead to effective heating and transport of plasma at the magnetopause [30,32, 33, 37]. Recent THEMIS spacecraft observations [35] show a direct evidence of a turbulent spectrum of KAWs at the magnetopause.

According to linear theory, when an MHD fast mode compressional wave propagates in an inhomogeneous plasma, it is coupled with the shear Alfvén wave at the local Alfvén resonance where $\omega=k_{\|} V_{A}$ [38-42], with $\omega$ being the wave frequency, $k_{\|}$the parallel wave number, and $V_{A}$ the Alfvén speed. In higher frequency cases, the Alfvén resonance condition is modified as $\omega^{2}=k_{\|}^{2} V_{A}^{2}\left(1-\omega^{2} / \Omega_{i}^{2}\right)^{2}$ due to the finite ion Larmor radius effects [43], where $\Omega_{i}$ is the ion gyrofrequency. While the MHD equations become singular at the Alfvén resonance point, in the kinetic description the wave coupling is through the mode conversion to kinetic Alfvén 
waves [31,32, 34,44]. In the magnetosphere, fast mode compressional waves originated from the foreshock region of the quasi-parallel bow shock $[45,46]$ frequently impinge onto the magnetopause, where the magnetic field increases and density decreases and there thus exists a sharp increase in the Alfvén velocity. The mode conversion may occur at the point in the magnetopause where the incident frequency matches the Alfvén resonance condition.

Although analytical solutions have been obtained for mode conversion at the magnetopause [32,34], there has been few kinetic simulation addressing the mode conversion in this region. Previously, we conducted a three-dimensional (3-D) global hybrid simulation of the dayside magnetosphere associated with a quasi-parallel bow shock [47]. It was found that as the compressional waves generated in the foreshock of the bow shock propagate to the magnetopause, they lead to strong surface perturbations and mode convert to shear Alfvén waves in the magnetosphere. At the magnetopause, wave energy builds up with wavelength $\lambda_{\perp} \sim \rho_{i}$, with a strong local enhancement of the parallel electric field. These waves excited in the magnetopause appear to be consistent with KAWs, but the wave mode and its formation process were difficult to identify in the global simulation. In order to identify the KAWs in the complicated global system, it is necessary to first understand the mode conversion process in a simple current sheet geometry.

In this paper, our recent two-dimensional (2-D) [48] and 3D hybrid simulations are shown for evolution of an incident compressional fast mode wave at the dayside magnetopause. The hybrid model solves fully kinetic equations governing the ions and a fluid model for electrons including electron pressure effects. The focus of this study will be on the identification of the mode conversion and the excitation and evolution of KAWs. For this study, we consider that locally the background magnetic field can be approximated by a slab geometry with one-dimensional variation normal to the boundary. We only show results of cases in which the wave vector $\boldsymbol{k}_{0}$ of the incident wave lies in the plane defined by the background magnetic field $\boldsymbol{B}_{0}$ (along $\boldsymbol{z}$ ) and the magnetopause normal (along $\boldsymbol{x}$ ).

The 2-D simulation results will be compared with linear theories of mode conversion $[32,34,44]$. Basic nonlinear physics of the mode conversion process will also be examined. More important physics for the plasma transport, however, lies beyond the 2-D limitation. The diffusion coefficient across a density gradient results from incoherent scattering from the perturbed velocity in the direction of the density gradient. Components of the perturbed drift velocities across the magnetopause are all proportional to the azimuthal mode number $k_{y}$, and the diffusion coefficient is proportional to $k_{y}^{2}[32,49]$. Fast waves generated in the magnetosheath most likely have global scale azimuthal mode numbers, so it is particularly important to examine whether small scale (large $k_{y}$ ) azimuthal modes can be generated in the 3-D mode conversion process. Although the background $B_{y}=0$ in the case shown in this paper, the 3-D physics is applicable to the generation of azimuthal wave numbers that is perpendicular to both $\boldsymbol{B}$ and $\boldsymbol{x}$ in the general cases of $B_{y} \neq 0$.

The outline of this manuscript is as follows. The simulation model is described in Section 1. In Section 2, we present the 2-D simulation results of linear and nonlinear properties of mode conversion process. The 3-D simulation results are presented in Section 3. A summary and discussion is given in Section 4.

\section{Hybrid simulation model}

In the calculation, ions are treated as fully kinetic particles moving in a self-consistent electromagnetic field, and electrons are treated as a corresponding massless fluid. Quasicharge neutrality is assumed.

The 2-D simulation [48] is performed in the $x z$ plane, with $x$ being the direction normal to the magnetopause and $z$ the direction of wave vector tangential to the magnetopause. Initially, the magnetopause current sheet in slab geometry is assumed to be centered at $x=0$ in the middle of the simulation domain, separating two uniform plasma regions of the magnetosheath $(x<0)$ with a high density and low magnetic field strength and magnetosphere $(x>0)$ with a low density and high magnetic field. The initial current sheet is assumed to be a tangential discontinuity, with the normal component of magnetic field $B_{x}=0$. For the cases shown in this paper, the magnetic fields in the magnetosheath and magnetosphere point along $z$ and are parallel to each other. Effects of the background magnetic field oblique to $z$ can be seen in Lin et al. [48].

In the calculation, the ion particles are advanced by the ion equation of motion:

$$
m_{i} \frac{\mathrm{d} \boldsymbol{v}_{i}}{d t}=e\left(\boldsymbol{E}+\boldsymbol{v}_{i} \times \boldsymbol{B}\right),
$$

where $\boldsymbol{v}_{i}$ is the ion particle velocity, $\boldsymbol{E}$ is the electric field, $\boldsymbol{B}$ is the magnetic field, $m_{i}$ is the ion mass, and $e$ is the elementary charge. The electric field can be obtained from the massless electron momentum equation:

$$
\boldsymbol{E}=-\boldsymbol{V}_{e} \times \boldsymbol{B}-(1 / N e) \nabla P_{e},
$$

where $V_{e}$ and $P_{e}$ are the flow velocity and thermal pressure of the electron fluid, and $N$ is the ion number density. The electron fluid is assumed to be isothermal, with temperature

$$
T_{e}=\mathrm{const}
$$

during the evolution, and thus $\nabla P_{e}=T_{e} \nabla N$. The electron flow speed is evaluated from Ampere's law,

$$
\boldsymbol{V}_{e}=\boldsymbol{V}_{i}-\frac{\nabla \times \boldsymbol{B}}{\mu_{0} N e} .
$$

The magnetic field is advanced in time from Faraday's law

$$
\frac{\partial \boldsymbol{B}}{\partial t}=-\nabla \times \boldsymbol{E} .
$$


Let the subscripts " $s$ " and " $m$ " represent the quantities in the magnetosheath and magnetosphere, respectively. The initial profile of the ion number density is given by

$$
N_{0}(x)=\frac{1}{2}\left(N_{m}+N_{s}\right)+\frac{1}{2}\left(N_{m}-N_{s}\right) \tanh \left(x / D_{0}\right),
$$

where $D_{0}$ is the half-width of the magnetopause current sheet. The initial ion temperature $T_{i 0}$ and the electron temperature $T_{e 0}$ are assumed to be uniform everywhere, while the ions are loaded with an isotropic, Maxwellian velocity distribution. For a given magnetosheath ion beta $\beta_{i s}$ and $T_{e 0} / T_{i 0}$, the initial magnetic field $B(x)$ is determined by the total pressure balance

$$
P_{0}(x)+B_{0}(x)^{2} / 2 \mu_{0}=\text { const }
$$

throughout the simulation domain, where the total thermal pressure $P_{0}=P_{i 0}+P_{e 0}$.

The ion number density in the magnetosheath is chosen to be $N_{s}=1000$ per cell and the number density in the magnetosphere is $N_{m}=N_{s} / 10=100$. The uniform grid size in the $x$ direction is chosen to be $\Delta x=0.5 d_{i s}$, where $d_{i s}$ is the ion skin depth in the magnetosheath, and the grid size in the $z$ direction is $\Delta z=2 d_{i s}$. The size of the simulation domain is chosen around $L_{x} \times L_{z}=200 d_{i s} \times 256 d_{i s}$. The time step is $\Delta t=0.05 \Omega_{s}^{-1}$, where $\Omega_{s}$ is the ion gyrofrequency in the magnetosheath.

Periodic boundary conditions are assumed at $z=0$ and $z=L_{z}$. Free boundary conditions are used at $x=L_{x} / 2$ on the magnetospheric side. The solar wind wave perturbations are imposed from the incoming boundary at $x=-L_{x} / 2$ in the magnetosheath and assumed to be a sinusoidal wave with a single frequency, $\omega=\omega_{0}$. For each case, the quantities $k_{z}$, $a \equiv \omega_{0} /\left(k_{\| 0} V_{A s}\right)$, and $\delta V_{i}$ of the incident wave are prescribed, where $k_{\| 0}$ is the initial parallel wave number, $V_{A s}$ is the magnetosheath Alfvén speed, and $\delta V_{i}$ is the wave amplitude in the flow velocity. The imposed incident wave is assumed to satisfy the MHD fast mode dispersion relation [50].

In the 3-D simulation, the initial incident wave is imposed exactly the same as that in the 2-D cases, propagating in the $x z$ plane. The additional grid size in $y$ is $\Delta y=d_{i s}$, and the periodic boundary conditions are assumed at the $y$-boundary surfaces. The grid dimensions $n_{x} \times n_{y} \times n_{z}=200 \times 65 \times 65$.

In the presentation below, the time is normalized to $\Omega_{s}^{-1}$. The magnetic field is expressed in units of $B_{s}$, the ion number density in units of $N_{s}$, and the temperature in units of $B_{s}^{2} /\left(\mu_{0} N_{s}\right)$. The velocity is normalized to the magnetosheath Alfven speed $V_{A s}$, and the spatial coordinates are normalized to $d_{i s}$.

\section{2-D simulation results}

The 2-D results of case 1 are shown below, in which $\beta_{i s}=0.5$, and $T_{e 0} / T_{i 0}=0.4$ for the background magnetic field and plasma, and $\omega_{0}=0.392, a=2.0$, and $\delta V_{i}=0.04$ in the incident compressional wave. The half-width of the magnetopause current layer is $D_{0}=7.5$. The Alfvén speed $V_{A}$ increases by nearly a factor of 4 through the magnetopause boundary, whereas the point with the Alfvén resonance condition is satisfied is at $x \simeq 53$. The incident fast wave propagates in the $x z$ plane with $\left(k_{x 0}, k_{y 0}, k_{z 0}\right)=(0.262,0,0.196)$.

Figure 1(a) depicts contours of magnetic field components $B_{x}$ and $B_{z}$, ion density $N$, ion flow component $V_{i x}$, and the $E_{y}$ component of electric field in partial domain around the magnetopause, obtained in case 1 at an early time $t=80$ when the incident compressional wave just reaches the edge of the magnetopause boundary near $x=-5$ from the left. At later time $t=240$, as shown in Figure 1(b) after the interaction of the incident wave with the magnetopause transition layer, reflected waves are also found on the magnetosheath side. Meanwhile, larger-amplitude, short wavelength waves have clearly formed in the transition layer, where the density and magnetic field have a large gradient. In these waves, the shear components $B_{y}$ and $V_{i y}, E_{x}$, and the parallel current density $J_{\|}$are well correlated. The parallel wavelength remains nearly unchanged, while the perpendicular wavelength has decreased significantly. On the right edge of the newly formed waves, the enhanced perpendicular wave vector $k_{x}$ has reversed direction from $k_{x}>0$ in the incident wave to $k_{x}<0$, as seen from the tilt angle of the wave fronts. These short wavelength waves radiate back to the magnetosheath, leading to structures with even larger $k_{\perp}=k_{x}$ in the boundary layer. Strongly enhanced parallel electric field $E_{\|}$is present in the resulting waves. In the case with a larger $\delta V_{i}=0.15$, strong enhancement in $T_{i}$ is obtained around the resonance point.

To illustrate the detailed structure of the short-wavelength waves generated in the magnetopause boundary layer, Figure 2 shows spatial cuts of the three magnetic field components and the parallel electric field $E_{\|}$along $x$ through the central region of the domain at $t=240$, where the background $B_{z}$ has a gradient, at $z \simeq 128$. In addition to the long-wavelength incident waves, coherent waves are seen to be excited apparently in $B_{y}$ and $E_{\|}$, with wavelength $\sim 3.15-7.35$ in the $x$ direction, corresponding to $k_{\perp} \rho_{i} \sim 0.4-1.0$. The vertical dashed line in Figure 2 indicates the position $x=X_{r}=4.0$. The strongest wave excitation is clearly coincident with the predicted Alfvén resonance position $X_{r}$, and upstream radiation of the waves is seen to some spatial extent. On the magnetospheric (right) side of $X_{r}$, the structure shows a pure spatial decay [48].

The excitation of waves at the Alfvén resonance point is consistent with the mode conversion to KAWs. Away from the Alfvén resonance point at $x=4.0$, waves satisfy the kinetic Alfvén wave dispersion relation. For example, near $x=0, \omega^{2} / k_{\|}^{2} V_{A}^{2} \simeq 1.78, k_{\perp} \rho_{i} \simeq 0.72$, and $b=k_{\perp}^{2} \rho_{i}^{2} \simeq 0.52$. The KAW dispersion relation requires

$\frac{\omega^{2}}{k_{\|}^{2} V_{A}^{2}}=1.78 \sim\left(\frac{b}{1-\Gamma_{0}(b)}+\frac{T_{e}}{T_{i}} b\right) \simeq\left(1+\left(1+\frac{T_{e}}{T_{i}}\right) b\right)=1.73$.

Therefore, the dispersion relation of KAWs is satisfied in 

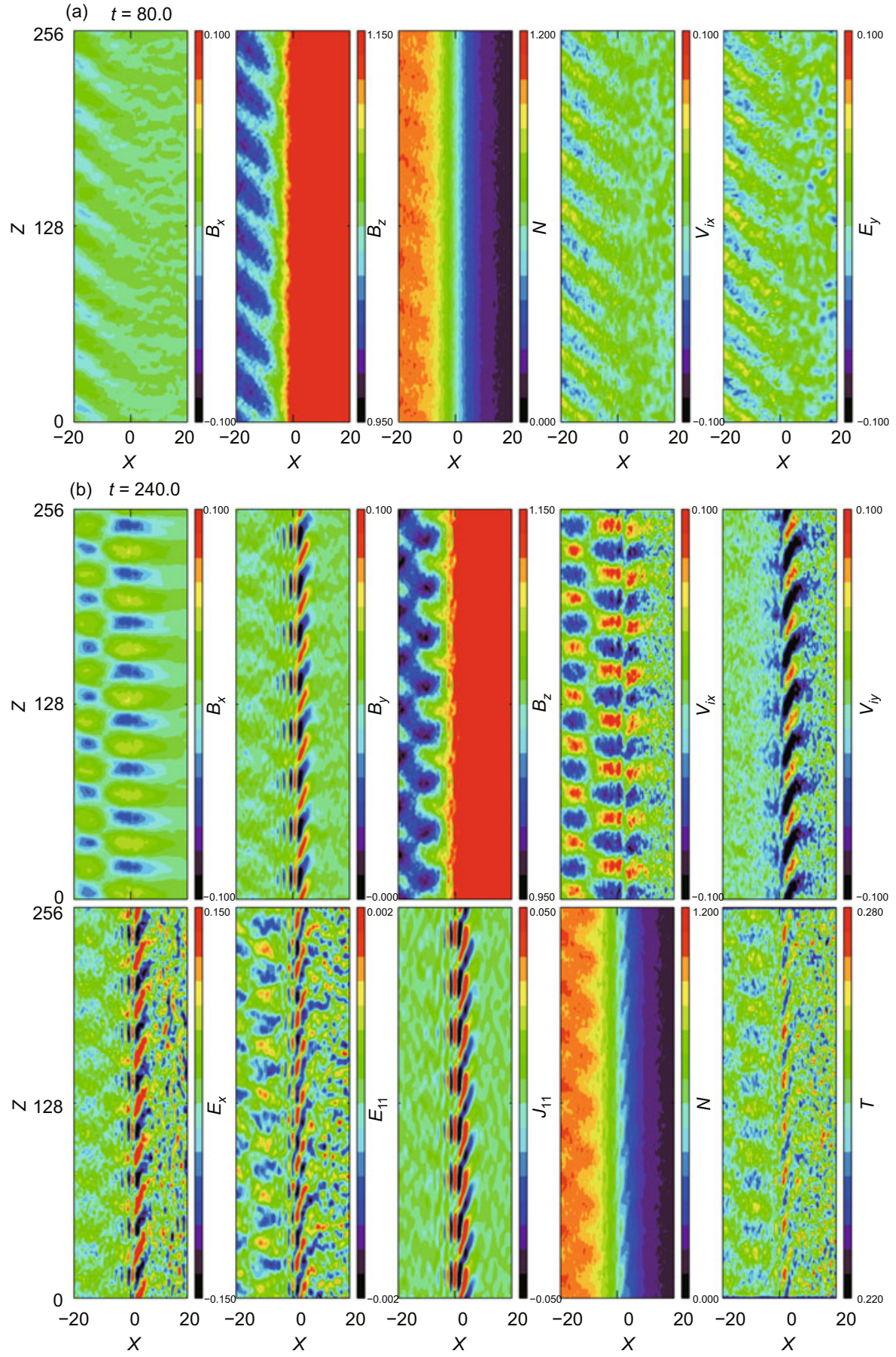

Figure 1 Contours of various quantities in partial domain around the magnetopause obtained in case 1 for (a) at an early time $t=80$ and (b) $t=240$. Short wavelength, coherent waves are generated by interaction between the incident wave and the magnetopause.

these excited waves in the magnetopause. On the left side of the resonance point, the Alfvén speed is smaller. The perpendicular wavelength appears shorter with enhanced $b=k_{\perp}^{2} \rho_{i}^{2}$. The dispersion relation for KAW is again nearly satisfied. The ratio $\left|\delta E_{\|}\right| /\left|\delta E_{x}\right|$ in the resulting KAW is found to be linearly proportional to $T_{e 0} / T_{i 0}$ [48], consistent with the linear two-fluid theory with gyrokinetic closure for the ion pressure [44, 51, 52].

It is found that for $\omega_{0}>0.1$, the mode conversion to KAWs takes place even for the case in which the incident compres- sional wave possesses no $\boldsymbol{k}_{\perp 0}$ transverse to both the magnetic field and the magnetopause normal, as seen in case 1 . This result is not expected based on the low-frequency theory of mode conversion [38-41], in which the compressional and transverse waves are completely decoupled in such case. Our results indicate that finite frequency effects can remove the de-coupling between the compressional and transverse waves as originally noted by [53]. In addition, the absorption varies significantly with $\omega_{0}$, peaking near $\omega_{0} \sim 0.3 \Omega_{s}$, consistent with a theoretical model [50] that solves an analytic 


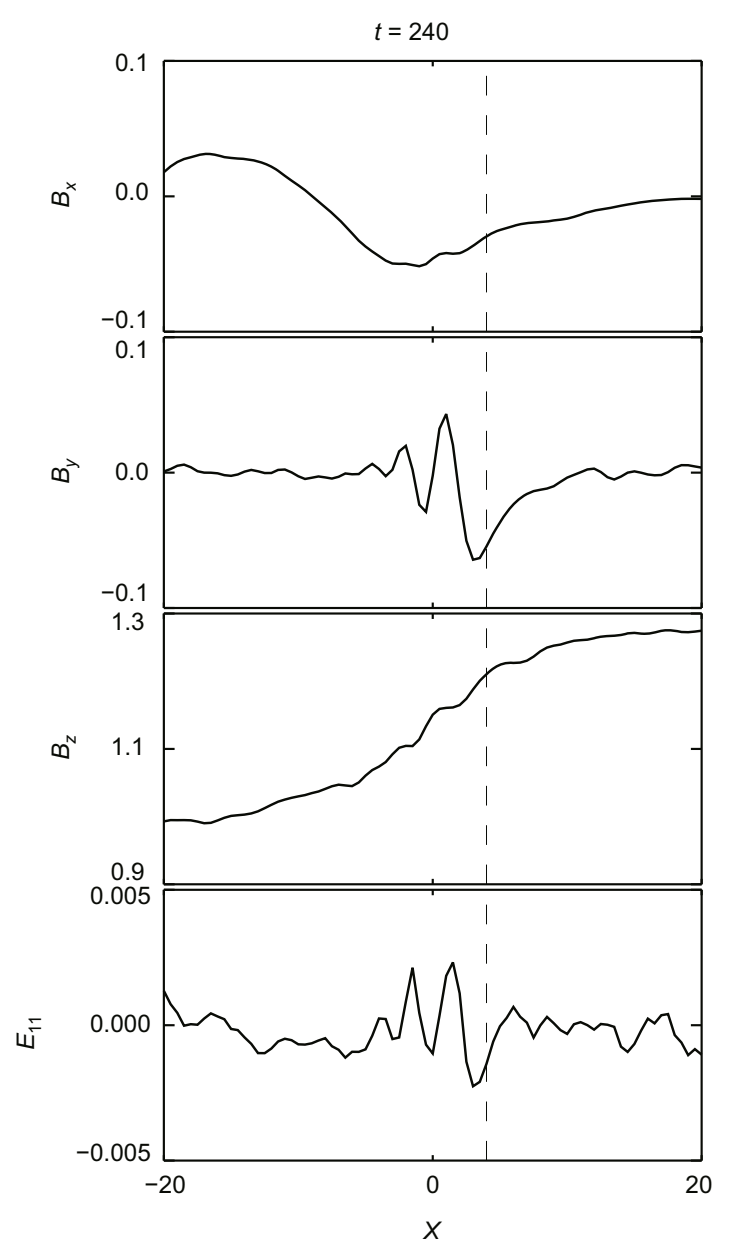

Figure 2 Spatial cuts of the magnetic field components and parallel electric field at $t=240$ along $x$ through the central region of the domain at $z \simeq 128$ in case 1 . The vertical dashed line indicates the resonance position $x=X_{r}=4.0$.

solution of the full fluid wave equations in a system containing an equilibrium structure of the magnetopause [48]. The efficiency of the mode conversion process can be greatly enhanced over the MHD limit and for a broad range of frequency can capture most of the wave power in the magnetopause boundary layer where it is converted into kinetic Alfvén waves. On the other hand, it has weak dependence on $D_{0}$ and $T_{e 0} / T_{i 0}$.

In addition to the linear properties discussed above, a number of interesting nonlinear properties are found in our simulation: passive generation of harmonics for weak nonlinear driving; wave breaking in the upstream compressional wave evidenced by upstream generation of nonlinear harmonics; and general broadening of the wave spectrum such that there is a broadening of the resonant region to encompass the entire Alfvén-cyclotron continuum across the magnetopause [48].

Besides the primary mode with $\omega=\omega_{0}$, multiple harmonics of $\omega_{0}$ are generated in the region of mode conversion, paired with harmonics of $k_{\| 0}$. The powers of these modes decrease with the order of the harmonics, with the primary harmonic mode $\omega=\omega_{0}$ being the strongest and dominant. For larger frequency and/or amplitude of the compressional wave driver, harmonics of $\omega_{0}$ can be generated in the incident compressional wave before it reaches the magnetopause, due to nonlinear steepening.

At finite frequency $\omega_{0} / \Omega_{i}$, the appearance of paired harmonics would alter the resonance location, occurring at $\omega=$ $k_{\|} V_{A}\left(1-\omega^{2} / \Omega_{i}^{2}\right)$, for the harmonic waves with $\omega_{0} / k_{\| 0}=$ $2 \omega_{0} / 2 k_{\| 0}=\cdots=n \omega / n k_{\| 0}=V_{A}$. Since higher harmonics would require a larger $V_{A}$ to satisfy the resonance condition, the nonlinear wave structure should shift toward the region of increasing $V_{A}$. In this manner the generation of harmonics near the fundamental resonance could lead to a spreading of the resonant wave structure in the direction of increasing $V_{A}$. However, the resonance condition itself may only be satisfied for a limited range of frequency which satisfies $n_{\|}^{2}=S$ between the primary resonance and the maximum Alfvén velocity on the right side of the domain. The broadening of the resonance is more pronounced at lower frequency.

In the simulation it also appears that nonlinear wave-wave interaction results in the broadening of the spectral bandwidth in $\omega$ and the appearance of large amplitude waves within the Alfvén continuum, as shown in Figure 3 for case 2 with $a=1.5, \omega_{0}=0.294, \beta_{i s}=0.5, T_{e 0} / T_{i 0}=0.4, \delta V_{i}=0.04$, $D_{0}=7.5$, and $k_{\| 0}=0.196$. Shown in Figure 3 are the spatial structures of $B_{y}$ as a function of $x$ around $z=128$ at $t=150$, 200 and 400. The vertical dashed lines indicate the resonance point at $x=X_{r}=-1.0$. The broadening of the Alfvén resonance region is evident at $t=150$, when the wavy structure of $B_{y}$ is seen throughout the region from $x=X_{r}$ to $x \simeq 9$ on its right, with a larger amplitude at $x=X_{r}$. At $t=200$, the

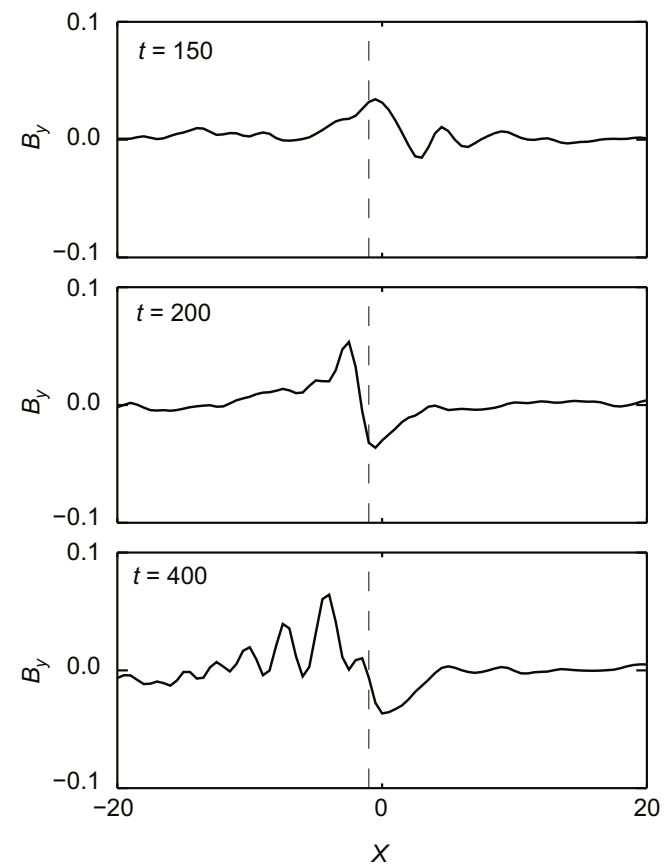

Figure 3 Spatial cuts of $B_{y}$ as a function of $x$ around $z=128$ at $t=150$, 200 and 400 show the broadening of the Alfvén resonance region in case 2. The vertical dashed lines indicate the resonance point at $x=X_{r}$. 
waves at $x=X_{r 1}$ become predominant, while weaker perturbations are still seen on its right. At $t=400$, the peak at $x=X_{r}$ has obviously shifted to the right, and the perturbations occupy a broad area. Again, strong KAWs with larger $k_{\perp}$ also radiate into the magnetosheath on the left side of $x=X_{r}$.

A close examination shows that on the right side of the resonance point $x=X_{r}$, the wave spectral width in $\omega$ is significantly broadened in the way that modes with frequencies other than $\omega_{0}$ are also excited at $k=k_{\| 0}$. Figure 4 depicts the powers of $B_{x}$ and $B_{y}$ in the $k_{\|}-\omega$ plane at $x=X_{r}=-1.0$ and $x=9.0$. Discrete harmonic modes $\left(k_{\| 0}, \omega_{0}\right)$ and $\left(2 k_{\| 0}, 2 \omega_{0}\right)$ are present clearly in all the three field components $\left(B_{z}\right.$ not shown) at the resonance point $x=-1.0$. At $x=9.0$ on the magnetospheric side, however, broad spectra with $\Delta \omega \simeq$ $0.9 \omega_{0}$ appear predominantly in $B_{x}$ and $B_{y}$ at $k_{\|}=k_{\| 0}$, in addition to the mode near $\left(k_{\| 0}, \omega_{0}\right)$. Continuous spectral curves are also shown across the $k_{\|}-\omega$ space, which are found to be the normal modes that also exist under a random perturbation of the boundary layer without the incident coherent waves and in principle could also be excited by the numerical noise. In fact, the dominant mode in $B_{y}$ appears at $\left(k_{\| 0}, 1.9 \omega_{0}\right)$, coincident with the curvy continuous spectrum at $k_{\| 0}$, whereas the power in $B_{x}$ is still dominated by the primary $\left(k_{\| 0}, \omega_{0}\right)$.

The broadening of the mode conversion region shown in Figure 3 may be associated with the broadening of $\omega$ spectrum at $k_{\|}=k_{\| 0}$. Due to the presence of compressional wave power in the broad band of wave frequency, the Alfvén res-

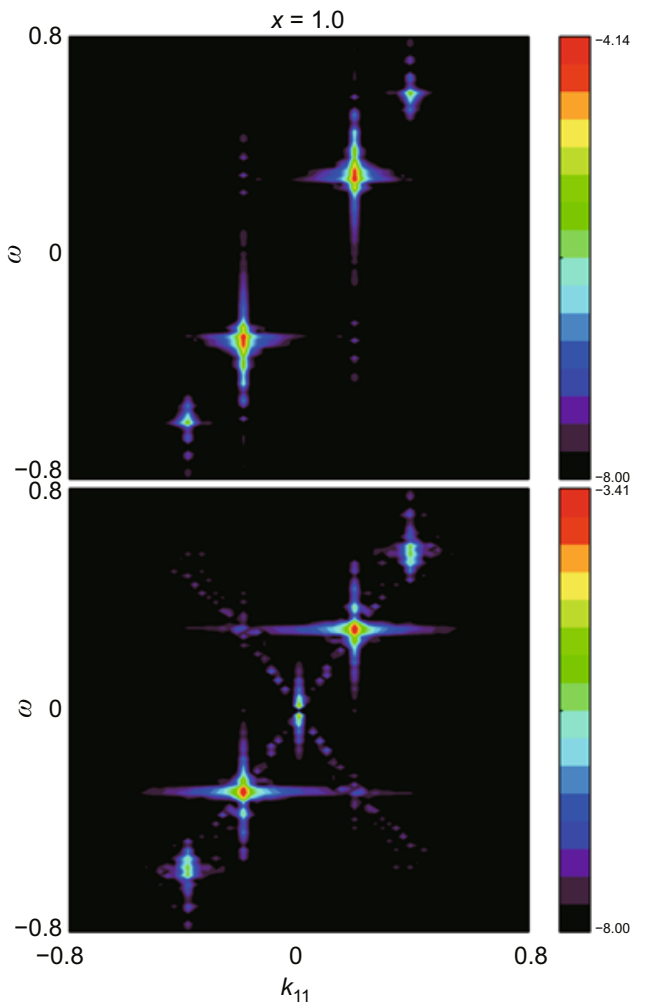

onance condition can be satisfied in a broad area of the density gradient layer, although the waves are weaker beyond the primary resonance point $x=X_{r}$. Such effect leads to the broadening of the resonance region, which imply that transport processes can occur over a wider region encompassing most of the magnetopause. Consequently, it is expected that diffusive processes would be more efficient in that they operate over a wider spatial region.

The excitation of modes in the continuum could result from a number of possible mechanisms. One possibility is that nonlinear effects broaden the wave spectrum near the primary resonance location. The frequency broadened waves could satisfy the resonance condition at a different location. Such waves could leak energy from the primary resonance location to the location where their shifted frequency matches the continuum frequency, leading to a pile up of energy at the shifted resonance location. Another possibility is that the waves are generated locally through nonlinear wave-wave interaction associated with a parametric excitation between the primary mode driver and the continuum at the shifted resonance location.

\section{3-D simulation results}

We now investigate the 3-D effects of mode conversion. The results of case 1 from the 3 -D hybrid simulation is discussed below.

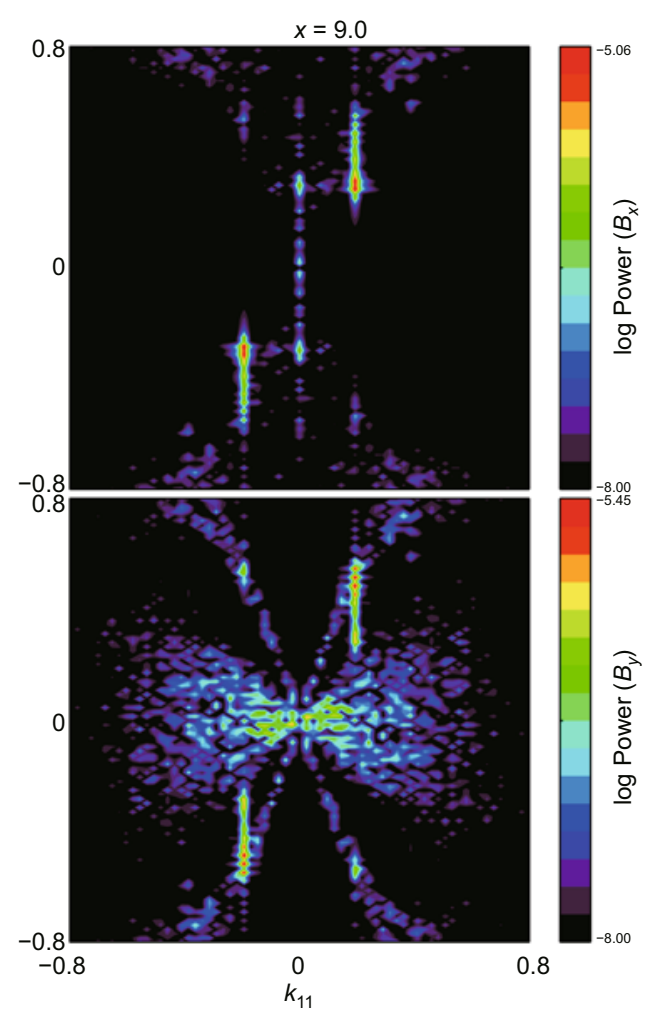

Figure 4 Powers of $B_{x}$ and $B_{y}$ in the $k_{\|}-\omega$ plane at $x=X_{r}=-1.0$ and $x=9.0$ obtained in case 2 . 
The resulting spatial structure in the $x z$ plane are similar to that of case 1. Larger-amplitude, short wavelength waves are excited in the transition layer between $x \simeq 47-60$ around the Alfvén resonance point. Similar to the 2-D results in Figure 1, these waves show characteristics of KAWs with large and broadband wave vectors $k_{x} \rho_{i} \sim 1$, dominated by perturbations in $B_{y}, E_{x}, E_{\|}$, and parallel current density $J_{\|}$.

Figure 5 shows the spatial structures in the $y z$ plane tangential to the magnetopause, at the resonant point $x=53$ at $t=200$. While $k_{y 0}=0$ in the incident wave, strong wave perturbations with large $k_{y}$ have clearly formed, as seen from the variations of all quantities. The excitation of the $k_{y}$ modes appears after the mode conversion from the compressional wave to the KAWs dominated by $k_{x}$ 's as discussed above. A spectral analysis in the $k_{y}-k_{\|}$plane for the wave structures at $x=53$ shows dominant wave powers at finite $k_{y} \sim 1$, corresponding to $k_{y} \rho_{i s} \sim 0.5$, where $\rho_{i s}$ is the ion Larmor radius in the magentosheath.

To understand the nonlinear physics of the 3-D mode con- version processes, we plot in Figure 6 the time evolution of the modes dominated by $k_{x}$ and modes dominated by $k_{y}$. Figure 6(a) shows the powers of $B_{x}$ (black), $E_{x}$ (green), $E_{y}$ (blue), and $E_{\|}$(red) of modes with $k_{x}=-1.0, k_{z}=0.196$, and all $k_{y}$ at $y=32$. It is seen that $E_{x}$ and $E_{\|}$grow from $t=40$, when the incident fast wave reaches the magnetopause boundary, while $B_{x}$ and $E_{y}$ show no growth, consistent with the presence of KAWs dominated by $k_{x}$. The powers are saturated in the second stage at $t \sim 80$. Finally, $E_{\|}$shows a growth again for $t>145$.

Overall, the first-stage growth of KAWs from $t=40$ to 80 is due to the linear physics of mode conversion from the fast wave to KAWs. The second and final stages of evolution for $t>80$ is due to the nonlinear physics in which the mode coupling to $k_{y}$ plays a significant role. The growth of small-scale $k_{y}$ modes after $t=80$ is illustrated in Figure 6(b), for modes with $k_{y}=-1.0, k_{z}=0.196$ and all $k_{x}$ at the resonance point $x=53$, corresponding to the dominant $k_{y}$ modes. In $t=80$ 145 , the strong growth in $B_{x}, E_{y}$ and $E_{\|}$is consistent with

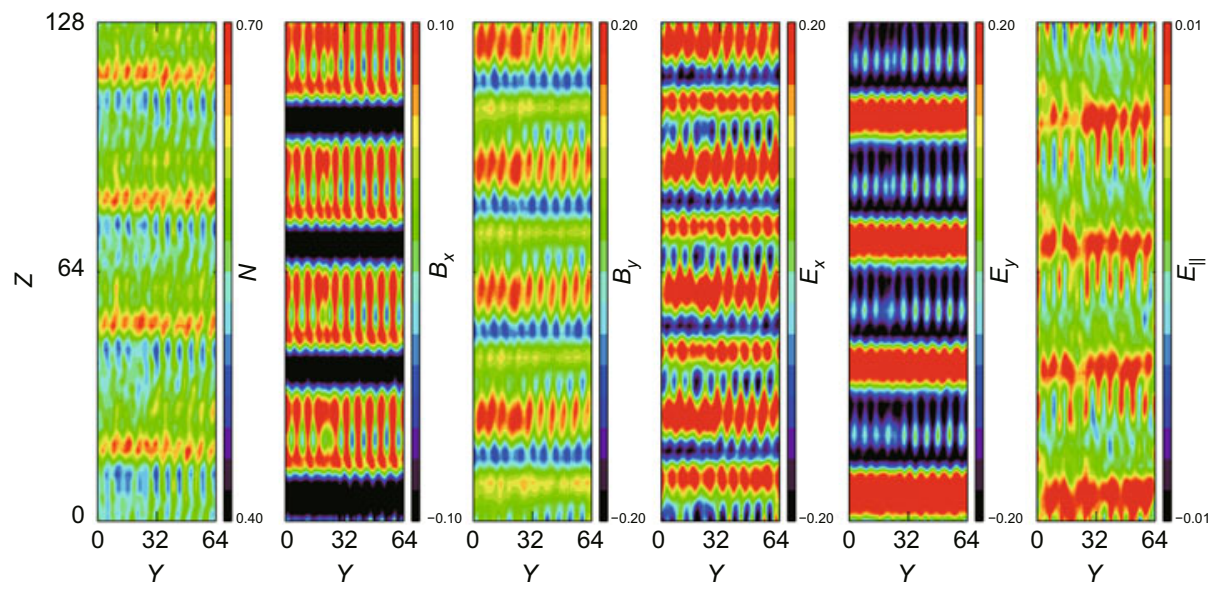

Figure 5 Contours of various quantities at $t=200$ in the $y z$ plane at the Alfvén resonant point $x=53$ obtained from the 3 -D simulation of mode conversion.
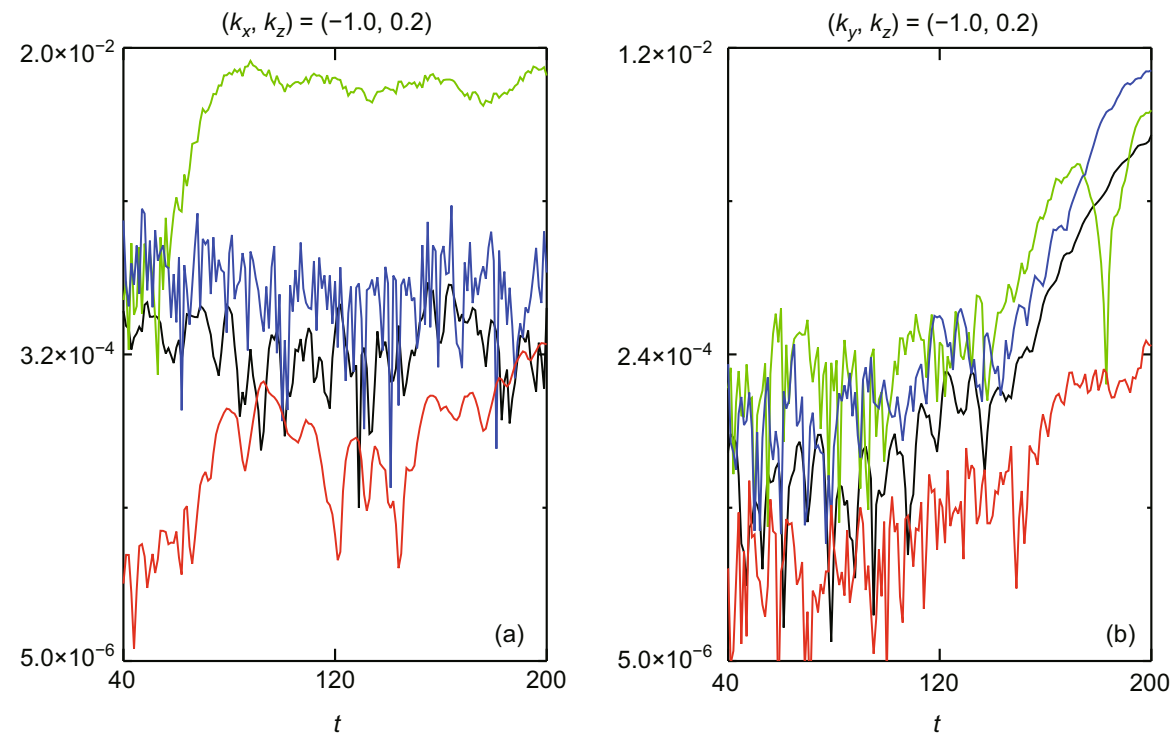

Figure 6 Time evolution of $B_{x}$ (black), $E_{x}$ (green), $E_{y}$ (blue) and $E_{\|}$(red) for the KAWs modes dominated by $k_{x}$ (a) at $y=32$ and for those dominated by $k_{y}$ (b) at the resonance point $x=53$ during the mode conversion. 
KAWs that are dominated by $k_{y}$, which nonlinearly co-exist with the KAWs dominated by $k_{x}$. Finally for $t>145$, the wave perturbations of $k_{x}$ and $k_{y}$ modes (displayed in Figure 6(b)) become isotropic with both $E_{x}$ and $E_{y}$ growing at the same rate.

The above results indicate a nonlinear decay of the primary KAWs dominated by $k_{x}$ to secondary KAWs dominated by $k_{y}$. Early theoretical work for cold plasmas $[54,55]$ $\left(\beta \sim\left(m_{e} / m_{i}\right)^{1 / 2} \ll 1\right.$, where $m_{e}$ and $m_{i}$ are the electron and ion masses, respectively) and a recent extension to finite $k_{\perp} \rho_{i}$ [56] found that a pump KAW can nonlinearly decay into an ion acoustic mode and a lower-sideband daughter KAW through a parametric instability. For a pump KAW propagating with $\boldsymbol{k}_{p}=\left(k_{x p}, 0, k_{z p}\right)$, where $z$ is the direction of the background magnetic field $\boldsymbol{B}_{0}$, the nonlinear coupling coefficient is proportional to $\Lambda=-i\left(c_{s}^{2} / 2 \Omega_{i}\right)\left(\boldsymbol{k}_{s} \times \boldsymbol{k}_{p}\right) \cdot \boldsymbol{e}_{z}$, where $\boldsymbol{k}_{s}=k_{s} \boldsymbol{e}_{y}$ is the wave vector of the ion acoustic mode. Since the pump mode has $k_{y p}=0$, the wave number matching condition results in that the daughter KAW must have a finite wave number $\boldsymbol{k}_{d}=-k_{s} \boldsymbol{e}_{y}$. A further simulation indeed shows that an initial KAW in the hot plasma of case 1 with $k_{y}=0$ decays through a parametric instability similar to that described by Hasegawa and Chen [54], accompanied by the growth of an ion acoustic wave and a daughter KAW, both with $\left|k_{y} \rho_{i}\right| \sim 1$.

\section{Summary}

In summary, our hybrid simulations have illustrated how an incident fast-mode compressional wave mode convert both linearly and nonlinearly to KAWs near the Alfvén resonance surface in the magnetopause boundary. The 2-D simulation shows that as the compressional wave reaches the magnetopause boundary from the magnetosheath, strong KAWs are excited at the location where the Alfvén resonance condition $\omega=k_{\|} V_{A}(x)\left(1-\omega^{2} / \Omega_{i}^{2}\right)$ is satisfied. The simulation results are compared with the linear theory of mode conversion. The efficiency of the mode conversion process can be greatly enhanced over the MHD limit and for a broad range of frequency. Unlike the prediction from low-frequency theory of the Alfvén resonance, KAWs are found to be generated also in cases in which the incident wave vector tangential to the magnetopause is parallel to the magnetic field, provided that the driver frequency $\omega_{0}>0.1 \Omega_{s}$, where $\Omega_{s}$ is the ion gyrofrequency in the magnetosheath.

In addition to the linear physics, several nonlinear properties of the mode conversion have been obtained. Harmonics of $\omega_{0}$ are found to be generated in the mode conversion process, in addition to the primary $\omega_{0}$ of the incident wave. Nonlinear wave-wave interaction that develops near the primary resonance point can lead to a broadening of the spectrum at the resonance location because the dispersion depends on frequency. In addition, on the high $V_{A}$ side of the resonance, peaks occur at the local continuum frequency having $k_{\|}=k_{\| 0}$ (as well as its harmonics in many cases). Regardless of the cause, we have found that nonlinear interactions driven by a monochromatic compressional wave driver lead to a broadened resonant region (basically exciting the entire continuum and not just the primary resonance) in a significantly different manner than the broadening that would result if the incident wave packet had a frequency spectrum with a spectral width.

In the 3-D case, our simulation shows a multiple-stage process of the excitation of KAWs. Following the first stage of the mode conversion to KAWs with large $k_{x} \rho_{i} \sim 1$ near the Alfvén resonance surface, which propagate in the same $k_{x}-k_{z}$ plane as the incident wave as shown in the 2-D cases, KAW modes with large $k_{y}$ 's are generated. The generation of the finite $k_{y}$ modes results from nonlinear parametric decay of the first-stage primary KAWs. The cases shown in this paper corresponds to the magnetopause under the condition with a northward IMF component. In this situation, mode conversion of compressional waves at the Alfvén velocity gradient quickly cascades to KAWs with large azimuthal (east-west) wavenumber $k_{y}$ in a 3-D manner, which may lead to massive transport at the magnetopause and provide a mechanism of global plasma entry into the plasma sheet $[32,35]$.

Computer resources were provided by NAS and ASC. This work was supported by NSFC grants (41028003 and 40890163), NSF grants (ATM-0852682 and ATM-0646442) and NASA grant NNX10AK97G to Auburn University, and at PPPL by NASA grants (NNG07EK69I, NNH07AF37I, NNH09AM53I, NNH09AK63I, and NNH11AQ46I), NSF grant ATM0902730, and DOE contract DE-AC02-09CH11466.

1 Fujimoto M. Plasma entry from the flanks of the near-Earth magnetotail. J Geophys Res, 1998, 103: 4391-4408

2 Phan T D, Lin R P, Fuselier S A, et al. Wind observations of mixed magnetosheath-plasma sheet ions deep inside the magnetosphere. J Geophys Res, 2000, 105: 5497-5506

3 Hasegawa H, Maezawa K, Mukai T, et al. Plasma entry across the distant tail magnetopause 2. Comparison between MHD theory and observation. J Geophys Res, 2002, 107: SMP 6-1-SMP 6-8

4 Hasegawa H, Maezawa K, Mukai T, et al. Plasma entry across the distant tail magnetopause 1. Global properties and IMF dependence. J Geophys Res, 2002, 107: SMP 9-1-SMP 9-16

5 Wing S, Newell P T. 2D plasma sheet ion density and temperature profiles for northward and southward IMF. Geophys Res Lett, 2002, 29: 1307

6 Olieroset M, Phan T D, Fujimoto M, et al. Spatial and temporal variations in the cold dense plasma sheet: Evidence for a low-latitude boundary layer source. Geophys Monograph, 2003, 133: 253-264

7 Hasegawa H, Fujimoto M, Maezawa K, et al. Geotail observations of the dayside outer boundary region: Interplanetary magnetic field control and dawn-dusk asymmetry. J Geophys Res, 2003, 108: SMP 9-1-SMP 9-16

8 Wing S, Newell P T. LLBL contribution to the plasma sheet ions. Geophys Monograph, 2003, 133: 273-282

9 Wang W X, Hahm T S, Lee W W, et al. Nonlocal properties of gyrokinetic turbulence and the role of $\mathrm{E} \times \mathrm{B}$ flow shear. Phys Plas, 2007, 14: 072306

10 Johnson J R, Wing S. Northward IMF plasma sheet entropies. J Geophys Res, 2009, 114: A00D08

11 Anderson R R, Harvey C C, Hoppe M M, et al. Plasma waves near the magnetopause. J Geophys Res, 1982, 87: 2087-2107

12 Tsurutani B T, Thorne R M. Diffusion processes in the magnetopause boundary layer. Geophys Res Lett, 1982, 9: 1247-1250 
13 Rezeau L, Perraut S, Roux A, et al. Electromagnetic fluctuations in the vicinity of the magnetopause. Geophys Res Lett, 1986, 13: 10931096

14 Labelle J, Treumann R A. Plasma waves at the dayside magnetopause. Space Sci Rev, 1988, 47: 175-202

15 Rezeau L, Morane A, Perraut S, et al. Characterization of Alfvenic fluctuations in the magnetopause boundary layer. J Geophys Res, 1989, 94: $101-110$

16 Engebretson M J, Lin N, Baumjohann W, et al. A comparison of ULF fluctuations in the solar wind, magnetosheath, and dayside magnetosphere: 1. Magnetosheath morphology. J Geophys Res, 1991, 96: 3441-3454

17 Engebretson M J, Cahill Jr L J, Arnoldy R L, et al. The role of the ionosphere in coupling upstream ULF wave power into the dayside magnetosphere. J Geophys Res, 1991, 96: 1527-1542

18 Lin N, Engebretson M J, McPherron R L, et al. A comparison of ULF fluctuations in the solar wind, magnetosheath, and dayside magnetosphere. II. Field and plasma conditions in the magnetosheath. J Geophys Res, 1991, 96: 3455-3464

19 Takahashi K, Sibeck D G, Newell P T, et al. ULF waves in the lowlatitude boundary layer and their relationship to magnetospheric pulsations: A multisatellite observation. J Geophys Res, 1991, 96: 95039519

20 Rezeau L, Roux A, Russell C T. Characterization of small-scale structures at the magnetopause from ISEE measurements. J Geophys Res, 1993, 98: 179-186

21 Song P, Russell C T, Fitzenreiter R J, et al. Structure and properties of the subsolar magnetopause for northward interplanetary magnetic field Multiple-instrument particle observations. J Geophys Res, 1993 98: 11319-11337

22 Song P, Russell C T, Strangeway R J, et al. Wave properties near the subsolar magnetopause-Pc 3-4 energy coupling for northward interplanetary magnetic field. J Geophys Res, 1993, 98: 187-196

23 Song P, Russell C T, Huang C Y. Wave properties near the subsolar magnetopause-Pc 1 waves in the sheath transition layer. J Geophys Res, 1993, 98: 5907-5923

24 Anderson B J, Fuselier S A, Gary S P, et al. Magnetic spectral signatures in the Earth's magnetosheath and plasma depletion layer. J Geophys Res, 1994, 99: 5877-5891

25 Song P. ISEE observations of the dayside magnetosheath. Adv Space Res, 1994, 14: 71-80

26 Song P, Russell C T, Gary S P. Identification of low-frequency fluctuations in the terrestrial magnetosheath. J Geophys Res, 1994, 99: 60116025

27 Phan T D, Paschmann G, Baumjohann W, et al. The magnetosheath region adjacent to the dayside magnetopause: AMPTE/IRM observations. J Geophys Res, 1994, 99: 121-141

28 Phan T D, Paschmann G, Sonnerup B U O. Low-latitude dayside magnetopause and boundary layer for high magnetic shear 2. Occurrence of magnetic reconnection. J Geophys Res, 1996, 101: 7817-7828

29 Phan T D, Larson D E, Lin R P, et al. The subsolar magnetosheath and magnetopause for high solar wind ram pressure: WIND observations. Geophys Res Lett, 1996, 23: 1279-1282

30 Hasegawa A, Mima K. Anomalous transport produced by kinetic Alfvén wave turbulence. J Geophys Res, 1978, 83: 1117-1123

31 Lee L C. Kinetic Alfvén Waves as a source of plasma transport at the dayside magnetopause. J Geophys Res, 1994, 99: 17405-17411

32 Johnson J R, Cheng C Z. Kinetic Alfvén waves and plasma transport at the magnetopause. Geophys Rev Lett, 1997, 24: 1423-1426
33 Chen L. Theory of plasma transport induced by low-frequency hydromagnetic waves. J Geophys Res, 1999, 104: 2421-2428

34 Johnson J R, Cheng C Z, Song P. Signatures of mode conversion and kinetic Alfvén waves at the magnetopause. Geophys Rev Lett, 2001, 28 227-230

35 Chaston C, Bonnell J, McFadden J P, et al. Turbulent heating and crossfield transport near the magnetopause from THEMIS. Geophys Rev Lett, 2008, 351: L17S08

36 Chaston C C, Wiber M, Mozer F S, et al. Mode conversion and anomalous transport in Kelvin-Helmholtz vortices and kinetic Alfvén waves at the Earth's magnetopause. Phys Rev Lett, 2007, 99: 175004

37 Johnson J R, Cheng C Z. Stochastic ion heating at the magnetopause due to kinetic Alfvén waves. Geophys Rev Lett, 2001, 28: 4421-4424

38 Tamao T. Transmission and coupling resonance of hydromagnetic disturbances in the non-uniform Earth's magnetosphere. Tohoku Geophys J, 1965, 17: 43-72

39 Uberoi C. Alfvén Waves in inhomogeneous magnetic fields. Phys Flu, 1972, 15: 1673-1675

40 Chen L, Hasegawa A. Plasma heating by spatial resonance of Alfvén wave. Phys Flu, 1974, 17: 1399-1403

41 Southwood D J. Some features of field line resonances in the magnetosphere. Planet Space Sci, 1974, 22: 483-491

42 Hasegawa A, Hsui K H, Assis A S. A theory of long period magnetic pulsations. III - Local field line oscillations. Geophys Res Lett, 1983 , 10: 765-767

43 Stix T H. Waves in Plasmas. New York: American Institute of Physics, 1992. 50-100

44 Hasegawa A, Liu C. Kinetic processes in plasma heating by resonan mode conversion of Alfvén wave. Phys Flu, 1976, 19: 1924-1934

45 Hoppe M M, Russell C T, Frank L A, et al. Upstream hydromagnetic waves and their association with backstreaming ion populations-ISEE and 2 observations. J Geophys Res, 1981, 86: 4471-4492

46 Hoppe M M, Russell C T. Plasma rest frame frequencies and polarizations of the low-frequency upstream waves - ISEE 1 and 2 observations. J Geophys Res, 1983, 88: 2021-2027

47 Lin Y, Wang X Y. Three-dimensional global hybrid simulation of dayside dynamics associated with the quasi-parallel bow shock. J Geophys Res, 2005, 110: A12216

48 Lin Y, Johnson J R, Wang X Y. Hybrid simulation of mode conversion at the magnetopause. J Geophys Res, 2010, 115: A04208

49 Chaston C C, Johnson J R, Wilber M, et al. Kinetic Alfvén Wave turbulence and transport through a reconnection diffusion region. Phys Rev Lett, 2009, 102: 015001

50 Streltsov A V. Plasma Waves. San Diego: Academic Press, 1989

51 Chaston C C, Lotko W, Johnson J R, et al. Small-scale, dispersive field line resonances in the hot magnetospheric plasma. J Geophys Res, 1998, 103: 26559-26572

52 Cheng C Z, Johnson J R. A kinetic-fluid model. J Geophys Res, 1999, 104: 413-428

53 Karney C F F, Perkins F W, Sun Y C. Alfvén resonance effects on magnetosonic modes in large tokamaks. Phys Rev Lett, 1979, 42: 16211624

54 Hasegawa A, Chen L. Parametric decay of "kinetic Alfvén wave" and its application to plasma heating. Phys Rev Lett, 1976, 36: 1362-1365

55 Frieman A, Chen L. Nonlinear gyrokinetic equations for low-frequency electromagnetic waves in general plasma equilibrium. Phys Flu, 1982, 25: 502-508

56 Chen L, Zonca F. Gyrokinetic theory of parametric decay of kinetic Alfvén waves. Europhys Lett, 2011, 36: 35001

Open Access This article is distributed under the terms of the Creative Commons Attribution License which permits any use, distribution, and reproduction in any medium, provided the original author(s) and source are credited. 